Canadian University Music Review

Canadian University Music Review

Revue de musique des universités canadiennes

\title{
Olivier Messiaen et le Québec : une présence et une influence déterminante sur la création musicale de l'après-guerre
}

\section{Jean Boivin}

Volume 17, numéro 1, 1996

URI : https://id.erudit.org/iderudit/1014694ar

DOI : https://doi.org/10.7202/1014694ar

Aller au sommaire du numéro

\section{Éditeur(s)}

Canadian University Music Society / Société de musique des universités canadiennes

\section{ISSN}

0710-0353 (imprimé)

2291-2436 (numérique)

Découvrir la revue

\section{Citer cet article}

Boivin, J. (1996). Olivier Messiaen et le Québec : une présence et une influence déterminante sur la création musicale de l'après-guerre. Canadian University Music Review / Revue de musique des universités canadiennes, 17(1), 72-97. https://doi.org/10.7202/1014694ar
Résumé de l'article

À sa classe du Conservatoire de Paris, Olivier Messiaen a enseigné l'harmonie, l'analyse musicale ou la composition à un nombre important de musiciens et de compositeurs canadiens, pour la plupart nés ou vivant au Québec. Sa musique et ses idées ont eu un impact réel sur la création musicale d'avant-garde au Québec ainsi que sur l'enseignement de la composition surtout au cours des 15 années qui ont suivi la Seconde Guerre mondiale. Plusieurs des élèves québécois de Messiaen (par exemple Serge Garant, Clermont Pépin et Gilles Tremblay), sont devenus à leur tour des pédagogues et ont contribué à faire connaître ses œuvres et sa pensée.
All Rights Reserved @ Canadian University Music Society / Société de musique des universités canadiennes, 1996
Ce document est protégé par la loi sur le droit d'auteur. L'utilisation des services d'Érudit (y compris la reproduction) est assujettie à sa politique d'utilisation que vous pouvez consulter en ligne.

https://apropos.erudit.org/fr/usagers/politique-dutilisation/ 


\title{
OLIVIER MESSIAEN ET LE QUÉBEC : UNE PRÉSENCE ET UNE INFLUENCE DÉTERMINANTE SUR LA CRÉATION MUSICALE DE L'APRÈS-GUERRE
}

\author{
Jean Boivin
}

Olivier Messiaen est venu trois fois au Québec ${ }^{1}$. Sa première visite remonte à août 1962, où il était venu à Montréal pour jouer en duo avec Yvonne Loriod, depuis peu son épouse, ses Visions de l'Amen pour deux pianos (1943). Le couple revient interpréter cette même œuvre en novembre 1970 , cette fois lors d'un concert de la Société de musique contemporaine du Québec (SMCQ), devant une salle comble et enthousiaste ${ }^{2}$. Maryvonne Kendergi, une amie intime du couple ${ }^{3}$, anime le lendemain une rencontre de plus de deux heures avec les étudiants et compositeurs de la Faculté de musique de l'Université de Montréal, auxquels se joignent les élèves de la classe de Gilles Tremblay au Conservatoire. Avant de répondre aux questions de l'assistance, Messiaen prononce une conférence intitulée « Rythme et ornithologie dans mes œuvres ». Quelques jours auparavant (les 27 et 28 octobre), le compositeur avait pu entendre une remarquable exécution de Chronochromie (1960) par l'Orchestre symphonique de Québec dirigé par Pierre Dervaux. Messiaen séjourne une dernière fois au Québec à l'automne 1978 afin d'assister aux trois concerts-hommage organisés conjointement par la SMCQ et l'Orchestre Symphonique de Montréal à l'occasion de son $70^{\mathrm{e}}$ anniversaire ${ }^{4}$. Une nouvelle rencontre avec le public, animée

1 Olivier Messiaen et Yvonne Loriod ont par ailleurs séjourné à Toronto en 1985 en qualité de membres du jury du Concours international Bach de piano, organisé au profit de la Fondation Glenn Gould.

2Le programme de ce concert du 5 novembre 1970, entièrement consacré à la musique de Messiaen, était complété par des extraits des Vingt Regards sur l'Enfant-Jésus pour piano (1944), interprétés par Yvonne Loriod.

3 Communicatrice bien connue des mélomanes québécois, Maryvonne Kendergi a largement contribué à la diffusion au Québec de la musique et des idées d'Olivier Messiaen (voir l'hommage qu'elle lui rendait dans le dossier « Olivier Messiaen, $70^{\circ}$ anniversaire », Variations 2, $\mathrm{n}^{\circ} 2$ [novembre 1978] :5, 16, 17) ainsi que dans le programme du concert-hommage rendu par la SMCQ à la même occasion $\left(n^{\circ} 109\right.$ [30 octobre 1978] : 5, 6, 31). Kendergi a réalisé sa première interview en 1957 avec le compositeur, interview diffusée dans le cadre de l'émission « Festivals européens » sur les ondes de Radio-Canada. Elle a interrogé à nouveau Messiaen une heure durant en 1962, cette fois en compagnie d'Yvonne Loriod. Kendergi s'est abondamment servie de ce matériel sonore dans ses cours d'histoire de la musique du $\mathrm{XX}^{\mathrm{e}}$ siècle à l'Université de Montréal. Des extraits de ces deux rencontres ont été réentendus sur les ondes de la radio française de Radio-Canada dans le cadre d'une émission consacrée à Olivier Messiaen et inscrite dans la série « Trésors d'archives de Maryvonne Kendergi » (31 janvier 1992, en reprise à l'été suivant) ; la réalisation de la série avait été confiée à l'auteur de ces lignes.

4Lors du concert donné par la SMCQ, on a pu entendre, outre quelques extraits des Vingt Regards 
par Kendergi, permet au compositeur d'expliquer sa démarche en présence des chefs d'orchestre Charles Dutoit et Serge Garant.

Le rayonnement de Messiaen au Québec est toutefois loin de se réduire à ses trois visites. Depuis le tournant des années 50, le concert, le disque et la radio de Radio-Canada ont permis au public intéressé de se familiariser avec les étapes d'une production qui compte parmi les plus importantes du $\mathrm{XX}^{\mathrm{e}}$ siècle. Mais c'est avant tout par le biais de son enseignement que Messiaen a pu exercer, dans l'immédiat après-guerre, une influence décisive au Québec.

\section{Un pédagogue entré dans la légende}

Messiaen a enseigné au Conservatoire national supérieur de musique de Paris du printemps 1941 à juin 1978, donc un total de 37 années, sans relâche. Il a été titulaire tour à tour d'une classe d'harmonie (1941-46), d'analyse musicale (1946-66) et enfin d'une classe de composition (1966-78). Nous avons décrit ailleurs les caractéristiques de la démarche pédagogique de ce professeur légendaire et avons tenté d'en cerner l'impact sur plusieurs générations de compositeurs $^{5}$. Il nous apparaît opportun d'examiner aujourd'hui la question d'un point de vue plus national.

Il s'avère en effet qu'un nombre important de Canadiens, pour la plupart francophones nés ou vivant au Québec, ont profité de l'accueil chaleureux et désintéressé réservé par ce peu banal professeur du Conservatoire aux auditeurs et aux visiteurs occasionnels. On trouvera en appendice un tableau où figurent, au meilleur de nos connaissances, les noms des élèves canadiens de Messiaen, dans l'ordre de leur arrivée à sa classe. Sauf précision contraire, il s'agit de compositeurs. Les Québécois dont les noms apparaissent en caractères gras retiendront particulièrement notre attention au cours des prochaines pages. Figurent aussi dans ce tableau les Canadiens qui ont assisté à des cours offerts par Messiaen non pas à Paris, mais plutôt à Tanglewood, à Darmstadt, à Buenos Aires ou à Avignon, villes où le compositeur français a été ponctuellement invité à donner des sessions de cours durant la période estivale 6 . Les noms des élèves de Messiaen d'origine étrangère qui ont ensuite résidé et enseigné au Canada sont inscrits en italiques ${ }^{7}$. Nous avons tenté de suggérer, par des traits verticaux ou des données figurant entre parenthèses, la durée approximative des séjours respectifs à la classe. Dans certains cas, l'expérience a été fort brève et se résume

pour piano solo, les Sept haïkaï (1962) et Couleurs de la Cité céleste (1963). La même semaine, les 31 octobre et $1^{\text {er }}$ novembre, l'œuvre pour orchestre Et exspecto resurrectionem mortuorum (1964) a été exécutée par l'OSM.

5 Jean Boivin, La classe de Messiaen, « Musique/Passé/Présent » (Paris : Christian Bourgois, 1995), 482 p.; version abrégée et remaniée d'une thèse de doctorat intitulée « La classe de Messiaen : historique, reconstitution, impact » (Université de Montréal, 1992), 755 p.

6Les sources biographiques se contredisent parfois à ce propos, mais il appert que Messiaen a participé à de telles sessions à Budapest et à Tanglewood en 1947; à Tanglewood à nouveau en 1949 ; à Darmstadt en 1949, en 1952, en 1953 et en 1961 ; à Sarrebruck en 1953 ; à Buenos Aires en 1963 ; et enfin à Avignon en 1987.

7Il est possible que d'autres compositeurs québécois aient visité la classe de Messiaen lors d'un séjour plus ou moins prolongé à Paris. Nous pensons par exemple à Michel-Georges Brégent et à Raynald Arsenault (tous deux décédés), dont les démarches présentaient quelques similitudes avec celle du compositeur français, notamment sur le plan spirituel. 
à quelques visites. À l'opposé, plusieurs peuvent être considérés comme de véritables élèves de Messiaen, qu'ils aient été inscrits officiellement ou non à sa classe ${ }^{8}$.

Cette liste laisse déjà entrevoir un important potentiel d'impact de la musique et de la pensée de Messiaen en sol canadien, surtout si l'on songe au fait qu'un bon nombre des élèves de Messiaen ont été à leur tour appelés à devenir des pédagogues. Nous avons choisi de nous limiter ici aux deux premières décennies de la carrière de pédagogue de Messiaen, de sa nomination au Conservatoire en 1941 , en pleine occupation allemande, jusqu'au tournant des années 60 . La présence québécoise chez Messiaen est alors particulièrement évidente. Certes, le compositeur français a pu exercer une influence sur d'autres musiciens canadiens, mais nous croyons que les compositeurs québécois se sont montrés généralement plus réceptifs à ses idées. En fait, comme on le verra, de nombreux facteurs en font un groupe à part. C'est pourquoi nous avons choisi de restreindre la présente étude aux seuls élèves québécois.

La période retenue présente aussi un intérêt particulier à nos yeux. C'est justement à la fin des années 40 et au cours des années 50 - soit durant le règne, à plusieurs égards inhibiteurs, de Maurice Duplessis (1936-39, 1944-59) que la musicologue Marie-Thérèse Lefebvre situe la naissance d'une réelle " modernité musicale » au Québec français9. De nombreux commentateurs issus de diverses disciplines s'entendent à remarquer durant cette période, dite de la « grande noirceur », une sorte de réveil artistique, une ébauche d'affirmation de la liberté de penser en matière de société et de culture. C'est l'époque du « Refus global » (1948) et des premières grandes toiles abstraites des Borduas et Riopelle, celle aussi de l'émancipation d'une littérature véritablement nationale, d'un théâtre et d'une chanson « à texte » bien ancrés dans la réalité du temps et du lieu ${ }^{10}$. Dans le domaine de la création musicale, on assiste également, de la part d'un petit nombre de jeunes loups, à une rupture avec le conformisme prudent véhiculé notamment par les institutions d'enseignement, depuis longtemps liées aux communautés religieuses. Un rejet assez net des modèles académiques accentuera bientôt l'écart entre, d'une part, ce groupe de compositeurs et, d'autre part, la critique et le grand public, jusqu'alors souverains juges de la valeur - voire de la validité - d'une œuvre musicale. On verra bientôt que Messiaen jouera dans ce processus d'éclosion un rôle de premier plan.

8La plupart des élèves québécois de Messiaen ne figurent pas sur ces listes puisqu'ils n'étaient présents chez Messiaen qu'à titre d'auditeurs ou de visiteurs occasionnels. Nos données sont tirées de sources biographiques diverses, dont l'Encyclopédie de la musique au Canada, $2^{\mathrm{e}}$ éd., éd. par Helmut Kallmann et Gilles Potvin (Montréal : Fides, 1993), ainsi que de témoignages directs ou indirects.

9 Voir notamment la première partie du livre de Marie-Thérèse Lefebvre intitulé Serge Garant et la révolution musicale au Québec (Montréal : Louise Courteau, 1986) ainsi que Paul-André Linteau, René Durocher, Jean-Claude Robert, Histoire du Québec contemporain, nouvelle édition refondue et mise à jour (Montréal : Boréal, 1989), vol. 2, Le Québec depuis 1930.

10Cette nouvelle culture nationale connaîtra bien sûr une explosion sans précédent lors de la « Révolution tranquille » qui succédera au régime duplessiste. 


\section{L'enseignement de la composition au Québec dans les années d'après-guerre}

Après la Seconde Guerre mondiale, l'éducation musicale supérieure au Québec est encore à l'état embryonnaire, surtout du côté francophone. Ceci est particulièrement sensible dans le domaine de la formation des compositeurs. En guise de points de repère, rappelons que le Conservatoire de musique de Montréal, calqué sur les conservatoires européens et plus spécifiquement sur celui de Paris, n'a été fondé qu'en $1942^{11}$; celui de Québec le sera deux ans plus tard et la Faculté de musique de l'Université de Montréal seulement en 1950. Auparavant, les apprentis-compositeurs étaient contraints d'acquérir les notions élémentaires d'harmonie et de contrepoint en privé, auprès des Claude Champagne, Alfred Laliberté, Léo-Pol Morin ou Gabriel Cusson. L'éducation musicale de niveau supérieur demeurait surtout l'apanage de la communauté anglophone, généralement mieux nantie et plus scolarisée ${ }^{12}$. Comme le soulignera Serge Garant, il ne pouvait guère être question pour les musiciens québécois francophones d'aller étudier aux États-Unis, les connaissances de l'anglais acquises à l'école étant généralement fort limitées ${ }^{13}$. C'est en France - la mère patrie comme on disait alors - que se rend l'élite des interprètes et les compositeurs ${ }^{14}$. Le Conservatoire national supérieur de Paris constitue l'objectif suprême. Plusieurs musiciens québécois de renom, tels le chef d'orchestre Jean-Marie Beaudet et le compositeur Claude Champagne, y ont fait des séjours d'études profitables. Parmi les élèves auxquels ce dernier pourra transmettre son amour indéfectible de la musique française, on compte Jocelyne Binet, Roger Matton, Pierre Mercure, François Morel, Serge Garant, Clermont Pépin et Gilles Tremblay. Tous, à l'exception de Pierre Mercure, seront bientôt des élèves de Messiaen à Paris ${ }^{15}$.

\section{Françoise Aubut, une pionnière à plus d'un titre}

L'organiste Françoise Aubut (1922-84) sera la première Canadienne à mériter pleinement le statut d'élève de Messiaen et ce, avant même le déclenchement de la Seconde Guerre mondiale, alors que le compositeur n'est connu en France que d'un petit nombre, essentiellement des amateurs d'orgue. Suivant le conseil

11 Les premiers cours ont été dispensés en janvier 1943 et la première année académique régulière a débuté en octobre de la même année.

12 À Montréal, l'Université McGill offrait un programme de composition depuis 1904 ! Claude Champagne y a enseigné l'écriture et la composition durant la décennie qui a précédé la Seconde Guerre mondiale, longtemps avant de détenir un poste officiel dans le secteur francophone (il allait devenir en 1942 assistant-directeur au nouveau Conservatoire de Montréal).

13 « Rencontre avec Serge Garant », interview de Maurice Fleuret, Cahiers canadiens de la musique, $\mathrm{n}^{\circ} 9$ (automne-hiver 1974) : 17, repris dans Lefebvre, Serge Garant et la révolution musicale au Québec, 75.

14Quelques Québécois francophones iront toutefois se perfectionner au Canada anglais ou aux États-Unis, certains par choix (tel Jean Papineau-Couture qui souhaite étudier avec Nadia Boulanger à Boston), d'autres par nécessité (tel Clermont Pépin, parce que la guerre fait rage en Europe).

15Quant à Jean-Marie Beaudet, il sera professeur entre 1947 et 1952 à l'École supérieure de musique d'Outremont (appelée communément École Vincent d'Indy), où il aura notamment comme élève Jocelyne Binet. C'est lui qui dirigea la création canadienne de la Turangalîla-Symphonie (1948) de Messiaen à Toronto, en 1964. 
de Nadia Boulanger rencontrée à Boston, Aubut part pour la France en 1938. La jeune musicienne n'a que 14 ans. Elle restera sept ans à Paris, jusqu'en 1945.

Inscrite à l'École normale de Paris dans les classes d'Alfred Cortot (piano) et de Nadia Boulanger (harmonie et « intelligence musicale ${ }^{16}$ ), Aubut entend vraisemblablement très tôt parler de Messiaen, titulaire des grandes orgues de l'église de La Trinité. Elle se joint rapidement à un petit groupe qui se retrouve fidèlement le dimanche pour écouter ce dernier improviser sur son instrument. Des réunions impromptues se tiennent à la tribune de la Trinité ou chez Messiaen lui-même. En ces temps troublés, les thèmes de l'art, de la foi et de la vie sont alors discutés librement, mais c'est bien sûr de musique - celle de Messiaen et des compositeurs qu'il apprécie - dont il est surtout question. Aubut est par ailleurs bientôt admise au Conservatoire de Paris dans les classes d'orgue de Marcel Dupré et de composition d'Henri Büsser. Ses études sont toutefois bientôt interrompues par la guerre. Elle connaît les privations de Paris occupée et, à titre de sujet britannique, est internée durant six mois dans un camp près de Besançon.

On sait qu'à compter du printemps 1940, Messiaen, après avoir été mobilisé dans le service infirmier, est lui aussi détenu dans un stalag. Il est libéré vers la fin de l'hiver 1941 et, après un court repos, est nommé titulaire d'une classe d'harmonie au Conservatoire. Françoise Aubut s'y inscrit à l'automne. Messiaen n'en continue pas moins de réunir en privé quelques musiciens intéressés par sa musique et ses recherches sur les modes, le plain-chant et les rythmes, éléments difficiles à intégrer dans un cours d'harmonie officiel destiné à préparer les élèves au concours de fin d'année. Aubut fait à nouveau partie de ce petit groupe de fidèles qui se réunissent souvent chez un compagnon de captivité de Messiaen, Guy-Bernard Delapierre. Dans la capitale occupée, les concerts sont rares et la musique nouvelle, considérée comme subversive, est vouée à la clandestinité. Plusieurs œuvres de Messiaen, dont des mélodies et le récent Quatuor pour la fin du Temps (1941), sont pourtant présentées à un public choisi, dans des salons privés. Une contre-culture musicale s'organise et Messiaen s'implique dans ces activités de survie artistique qui préparent l'élan créatif intense de la Libération. Aubut en témoigne :

À sa classe du Conservatoire de Paris ainsi qu'à ses cours chez Bernard Delapierre (rencontres qui réunissaient des musiciens tels que Loriod, Boulez, etc....) [Messiaen] parvenait à nous faire réaliser cet équilibre très rare entre l'instinct et la raison, l'élan et le contrôle ; il symbolisait pour nous l'indépendance et la liberté d'expression; en un mot il était incorruptible ${ }^{17}$.

Jusqu'en 1944, le nom d'Aubut figure sur les listes d'inscription à la classe d'harmonie de Messiaen. Son séjour au Conservatoire de Paris est couronné

16L'expression « intelligence musicale » est de Françoise Aubut et est tirée d'une interview qu'elle accordait en 1984, peu avant son décès, à Renée Larochelle pour l'émission « Les musiciens par eux-mêmes », à la radio FM de Radio-Canada.

$17 \mathrm{SMCQ}$, programme-hommage à Olivier Messiaen à l'occasion de son $70^{\mathrm{e}}$ anniversaire, $\mathrm{n}^{\mathrm{o}} 109(30$ octobre 1978) : 18 . 
cette même année d'une série de premiers prix : harmonie, contrepoint et fugue, histoire de la musique et, finalement, le grand prix d'improvisation à l'orgue, remis pour la première fois à une élève canadienne.

Dès son retour à Montréal, en 1945, Françoise Aubut défend avec ardeur la musique d'orgue de Marcel Dupré ainsi que celle de Messiaen, totalement inconnue au Québec et d'une surprenante modernité pour le public largement conservateur des concerts sacrés. Sa vie durant, elle voit en ce dernier compositeur « l'un des plus grands, peut-être même plus grand que $\mathrm{Bach}^{18}$. " À la fréquentation des Messiaen, Boulez, Loriod et autres succède toutefois la morne grisaille musicale montréalaise où, comme le souligne à juste titre la muséologue Denise Leclerc, « les préoccupations culturelles [...] n'occupaient qu'une modeste place dans le système de valeurs de la société d'après-guerre, davantage fascinée par les changements [d'ordre moral et surtout économique] qui affectaient son mode de vie ${ }^{19}$. » Pour Aubut, comme pour d'autres, le décalage est brutal : «Ici, la musique sérielle était une nouveauté, alors qu'elle était déjà dépassée là-bas ${ }^{20}$. » En tant que compositrice (car elle compose beaucoup à cette époque), elle traverse une profonde crise esthétique. Après quelques collaborations avec l'Office national du film, elle abandonne peu à peu l'écriture de création ${ }^{21}$.

Les connaissances acquises à Paris viendront heureusement nourrir les activités d'enseignement de cette musicienne accomplie. Peu après son retour, en 1946, elle est nommée professeure d'orgue au Conservatoire de Montréal, poste qu'elle occupera jusqu'en 1966. À compter de 1950, elle enseigne parallèlement l'orgue et l'improvisation à la toute nouvelle Faculté de musique de l'Université de Montréal et se joint l'année suivante au corps professoral de l'École supérieure de musique d'Outremont (École Vincent d'Indy) ${ }^{22}$. Aubut se fait par ailleurs un devoir de retourner en France aussi souvent que possible et de rendre visite à Messiaen auquel elle demeure très attachée. De son côté, il est certain que Messiaen l'appréciait et lui accordait toute sa confiance puisqu'il lui a demandé de le remplacer en 1948 à l'orgue de la Trinité alors que lui-même

18Extrait de l'interview accordée par Françoise Aubut pour l'émission « Les musiciens par euxmêmes », radio FM de Radio-Canada, 1984.

19Denise Leclerc, La crise de l'abstraction au Canada : les années 1950 (Ottawa : Musée des Beaux-Arts du Canada, 1992), 35.

20 Interview accordée par Françoise Aubut pour l'émission "Les musiciens par eux-mêmes », radio FM de Radio-Canada, 1984. L'allusion au sérialisme doit ici être comprise comme un point de repère historique plutôt que comme une référence directe aux idées de Messiaen ; celui-ci, tout en prônant une structuration de tous les aspects de l'inspiration musicale, gardait ses distances vis-à-vis des Viennois, et surtout de Schönberg dont il déplorait le conservatisme rythmique. Qui plus est, le système de composition avec 12 sons faisait fi selon lui du phénomène de la résonance harmonique naturelle, à la base de son propre langage musical. À ce propos, voir par exemple Messiaen, Musique et couleur, nouveaux entretiens avec Claude Samuel (Paris : Belfond, 1986), 53-55.

21 La carrière de Françoise Aubut a également été freinée par une santé fragile et surtout par un mariage malheureux, notamment sur le plan artistique.

22On compte parmi ses élèves Denis Regnaud (organiste, producteur puis chef des émissions musicales radio au réseau FM de Radio-Canada de 1987 à 1994), Françoys Bernier (chef d'orchestre, qui dirigera les Trois petites Liturgies de la Présence Divine de Messiaen à Québec en décembre 1965), Raynald Arsenault (organiste et compositeur) ainsi que Micheline Coulombe Saint-Marcoux (compositrice). 
effectuait une tournée aux États-Unis ${ }^{23}$. C'est lors d'un passage à la classe de Messiaen, à l'hiver 1952, qu'Aubut fera la connaissance de Karlheinz Stockhausen.

La vie musicale parisienne est à cette époque particulièrement bouillonnante et plusieurs œuvres importantes de Messiaen, comme les Trois petites Liturgies sur la Présence Divine (1944) ou le monumental cycle pour piano Vingt Regards sur l'Enfant-Jésus (1944), sont découvertes par un public d'autant plus réceptif qu'il avait été durement sevré par les années de guerre. La nouveauté du langage musical de ce jeune compositeur mystique, épris de couleurs et de chants d'oiseaux, attirera l'attention des mélomanes, mais jouera aussi contre lui. La presse sera souvent très dure à son égard et les membres de l'Institut, craignant son influence sur la jeune musique française, ne lui accorderont pas le poste devenu vacant de professeur de composition au Conservatoire. On crée plutôt en 1946 une classe d'analyse musicale qui va, contre toute attente, connaître une vive popularité auprès des jeunes créateurs aventureux et avoir l'importance historique que l'on sait. Une fois Françoise Aubut revenue de ce premier séjour à Paris, ses compatriotes Jocelyne Binet, Sylvio Lacharité, Serge Garant, Clermont Pépin et Roger Matton vont prendre le relais et participer, au tournant des années 50 , à cette nouvelle classe d'analyse.

\section{Jocelyne Binet, active dans l'ombre}

Avec Françoise Aubut, Jocelyne Binet (1923-68) a été l'une des toute premières compositrices du Québec. Il est regrettable que la documentation la concernant soit si lacunaire ${ }^{24}$. Après des études à l'École Vincent d'Indy auprès, notamment, de Claude Champagne, elle entreprend en 1948 un voyage d'études à Paris. Elle est admise, possiblement cette même année, au Conservatoire de Paris dans les classes de fugue et de contrepoint de Noël Gallon tout en poursuivant l'étude de la composition auprès de Tony Aubin et d'Arthur Honegger, ce dernier titulaire d'une classe à l'École normale. Il est difficile de déterminer avec précision quand et combien de temps Binet a été l'élève de Messiaen, mais nous croyons pouvoir situer cette expérience vers la fin de son séjour parisien, au cours de l'année académique 1950-5125.

Lorsqu'elle se rend à Paris, la jeune musicienne québécoise a l'ambition de faire de la composition une carrière. À son retour au pays, en 1951, les réalités financières et sociales ont raison de ce projet, difficilement réalisable pour une femme à l'époque. Elle s'oriente, tout comme Aubut, vers le professorat. Elle enseigne les techniques d'écriture et l'analyse formelle en privé ou à la licence et au baccalauréat ${ }^{26}$. Quelques notes de sa main, conservées aux Archives

23Lorsque nous avons interrogé Messiaen en 1987, il se souvenait très bien de Françoise Aubut ainsi que de quelques autres québécois dont il sera question plus bas, par exemple Sylvio Lacharité, Gilles Tremblay, Bruce Mather et Jacques Hétu.

24 Au sujet de Jocelyne Binet ainsi que de Françoise Aubut, on pourra relire les quelques notes rassemblées par Marie-Thérèse Lefebvre dans La création musicale des femmes au Québec (Montréal : Remue-ménage, 1991), 71-75.

25Le nom de Binet ne figure pas sur les listes officielles d'inscription à la classe de Messiaen.

26Binet fera partie du corps professoral de l'École Vincent d'Indy (1952-58?), du Conservatoire de musique de Montréal (1957-59) et plus spécialement de l'École de musique de l'Université Laval 
nationale du Québec à Québec, montrent que les connaissances acquises chez Messiaen pouvaient se transmettre à ses élèves ${ }^{27}$.

\section{Sylvio Lacharité, un nouveau disciple}

Originaire de Sherbrooke, Sylvio Lacharité (1914-83) obtient en 1949 une bourse du gouvernement du Québec lui permettant de compléter ses études musicales à Paris. Celui que Marie-Thérèse Lefebvre décrit comme un «homme de grande culture ${ }^{28}$ a alors 35 ans et se voue depuis plusieurs années à la direction d'orchestre ${ }^{29}$. Il s'embarque pour l'Europe le 10 février 1950 et séjournera à Paris un peu plus de deux ans. En sa qualité d'élève de Pierre Monteux ${ }^{30}$, le jeune musicien a déjà tissé de nombreux liens dans les milieux musicaux canadien, européen et américain ; il n'est toutefois pas certain qu'il ait entendu parler de Messiaen avant son arrivée à Paris ${ }^{31}$.

À compter du printemps 1950, Lacharité reçoit des leçons de contrepoint d'Andrée Vaurabourg-Honegger, la femme du célèbre compositeur et l'une des pédagogues les plus respectées de la capitale française. Il a l'intention de renouveler le contact avec Darius Milhaud qu'il avait connu à Aspen, au Colorado, et qui est titulaire d'une classe de composition au Conservatoire de Paris $^{32}$. Dans une lettre datée de la fin de juin et adressée à sa sœur Jeannette, Lacharité mentionne pour la première fois le nom de Messiaen. Oubliant son premier projet, il devient rapidement un auditeur très assidu de la " classe d'esthétique et d'analyse musicale », comme elle est baptisée alors ${ }^{33}$.

(1957-68). Serge Garant et Gilles Tremblay figurent au nombre de ses élèves en contrepoint.

27Les notes, succinctes, concernent essentiellement la forme sonate ; elles contiennent des remarques sur Beethoven, Mozart et Debussy qu'il est possible de relier à des propos tenus en classe par Messiaen (Fonds Jocelyne Binet, Archives nationales du Québec à Québec, document P 442).

28Lefebvre, Serge Garant et la révolution musicale au Québec, 25. La formation " classique 》 de Lacharité ressort d'ailleurs nettement des documents regroupés aux Archives nationales du Québec à Sherbrooke (Fonds Sylvio Lacharité).

29Lacharité avait fondé en 1939 l'Orchestre symphonique de Sherbrooke, l'un des plus anciens en sol québécois. Voir André Désilets, édit., La vie musicale à Sherbrooke, 1820-1989 (Sherbrooke : Société d'histoire de Sherbrooke, 1989), 61-65.

30Le créateur de Daphnis et Chloé et du Sacre du printemps enseignait la direction d'orchestre à Hancock, dans le Maine, où il avait fondé une école. Lacharité y a séjourné huit étés (1944-49, 1953-54).

31 Rappelons que Messiaen avait séjourné dans le Maine en 1947 et en 1949. À l'été 1947, Serge Koussevitzky dirige L'Ascension pour orchestre (1933) au festival de Tanglewood devant une foule imposante ; il s'agirait du début de la carrière américaine de Messiaen qui aurait été à cette occasion invité à donner une série de cours de composition (Messiaen, Musique et couleur, nouveaux entretiens avec Claude Samuel, 170-71). Le 2 décembre 1949, à Boston, le compositeur français assiste à la création de sa monumentale Turangalîla-Symphonie (1948), l'orchestre étant placé sous la direction du jeune Leonard Bernstein. Il donne à nouveau une série de cours, auxquels assistent les Canadiens Harry Freedman et Robert Turner.

32Il s'agit de la classe qui avait été refusée à Messiaen en 1946.

33Dans ses lettres à sa sœur, Lacharité parlera tout au long de son séjour de ses deux professeurs, Andrée Vaurabourg-Honegger et Messiaen. Les cours donnés par Messiaen, ainsi que d'autres pris à l'occasion à la Sorbonne (au département d'histoire de l'art), le tiennent fort occupé, sans parler des innombrables concerts, opéras et pièces de thêâtre auxquels il assiste, dans les limites de ce que lui permet son modeste budget (Fonds Sylvio Lacharité, Archives nationales du Québec à Sherbrooke, notamment la lettre du 28 juin 1950). Remarquons que Lacharité est alors aussi en contact épistolaire occasionnel avec Roger Matton, un futur élève de Messiaen. 
Les données rassemblées au cours de l'enquête que nous avons menée auprès d'anciens élèves de Messiaen nous permettent de repérer, parmi la douzaine de boîtes de partitions annotées conservées dans le Fonds Sylvio Lacharité, les œuvres qui ont été analysées par le pédagogue français durant la période où Lacharité séjourne à sa classe. Retenons quelques concertos pour piano de Mozart (KV 466 et 488), deux sonates pour piano de Beethoven (opp. 13 et 80), des études de Chopin, plusieurs Préludes de Debussy, des extraits de l'opéra Pelléas et Mélisande du même compositeur, le Wozzeck de Berg, la Deuxième sonate de Boulez, etc. Messiaen aurait aussi analysé au profit de ses élèves ses Vingt Regards sur l'Enfant-Jésus, ses Visions de l'Amen, ses Études de rythme (1949-50) et sa Messe de la Pentecôte (1950). Lorsqu'il traite de son travail personnel de création, le Maître ne dissimule rien de sa technique et parle longuement des modes à transposition limitée, des rythmes non rétrogradables, des mètres grecs et des deçî-talâs hindous (deux systèmes fort anciens à la base de son langage rythmique personnel) et ce, sans jamais vouloir imposer son esthétique ni son langage à quiconque.

Tout au long de sa fructueuse carrière au Québec, Lacharité s'est plu à se décrire comme un disciple de Monteux et de Messiaen ; il appert que ces deux personnalités ont laissé une empreinte profonde sur l'ensemble des activités entreprises par le musicien, qu'il s'agisse de direction d'orchestre (à Sherbrooke, à Québec et à Montréal), de travaux d'écriture ou d'arrangement (car il est aussi compositeur à ses heures), d'enseignement (il donnera notamment des cours d'analyse musicale et esthétique) ou de fonctions administratives (il sera successivement directeur des conservatoires de Québec et de Chicoutimi). Lacharité a acquis au fil des ans une quantité impressionnante d'œuvres de Messiaen en partition, notamment la Turangalîla-Symphonie et le Réveil des oiseaux pour piano et orchestre (1952-53), ainsi qu'une bonne part des pages écrites pour orgue. Dans l'hommage qu'il a rendu à Messiaen en 1978 à l'occasion de son $70^{\mathrm{e}}$ anniversaire ${ }^{34}$, c'est essentiellement à la dimension spirituelle et théologique de l'homme et de l'œuvre que s'est arrêté son ancien élève, lui-même profondément croyant ${ }^{35}$. Et malgré sa discrétion à cet égard, Lacharité devait se révéler un chaînon important de ce que nous pourrions appeler la « filière québécoise » des élèves de Messiaen puisqu'il passera directement le flambeau à l'un de ses jeunes élèves de piano à Sherbrooke, Serge Garant.

34SMCQ, programme-hommage à Olivier Messiaen, n 109 (30 octobre 1978) : 27.

$35 \mathrm{Il}$ est fort probable que les solides convictions religieuses de Messiaen aient pu ajouter à l'attrait éprouvé pour sa musique et son enseignement par plusieurs musiciens québécois de cette génération, éduqués pour la plupart par des membres de communautés religieuses, dans une société où l'Église catholique exerçait un contrôle indéniable sur les façons d'être et de penser. De même, cet aspect de la réputation du compositeur et pédagogue a sans doute rassuré les proches de ces jeunes musiciens canadiens-français qui avaient choisi d'affronter les difficultés - réelles à l'époque qui nous intéresse - d'un séjour d'études prolongé sur l'ancien continent. Cette question mériterait d'être explorée davantage. 


\section{Serge Garant et la découverte d'un nouvel univers sonore}

Avant son départ pour l'Europe, Lacharité a eu l'occasion d'enseigner des rudiments de piano à Serge Garant, son cadet de 15 ans $^{36}$. Il dirige également l'orchestre dans lequel Garant joue de la clarinette et l'initie même aux trésors de la peinture ainsi qu'à la poésie française et québécoise. En 1948, Garant se rend à Montréal pour, d'une part, poursuivre son apprentissage du piano auprès de la renommée pédagogue Yvonne Hubert et, d' autre part, entreprendre l'étude de la composition, qui l'attire avec force ${ }^{37}$. Une fois installé à Paris, Garant éprouve de plus en plus l'envie d'élargir lui aussi ses horizons. Il a déjà découvert dans les fonds de tiroir d'un marchand de Sherbrooke des pages de Schönberg et de Webern et a compris que la musique peut être " autre chose " que celle qu'il entend au Québec. Après avoir mis amis, parents et mécènes à contribution, il s'embarque le 3 octobre 1951 pour la France. Il se rend d'emblée chez Messiaen, auquel Lacharité le présente et le recommande.

Aux côtés de son ancien maître et ami, Garant étudie des partitions aussi nouvelles et riches que le Wozzeck et la Suite lyrique de Berg, ainsi qu'une série d'œuvres marquantes de Messiaen, les Vingt Regards sur l'Enfant-Jésus, les quatre Études de rythme pour piano (dont le fameux Mode de valeurs et d'intensités), la Messe de la Pentecôte et le Livre d'orgue (1951) ${ }^{38}$. "C'était une découverte absolue, de déclarer Garant, une découverte totale. J'apprenais tout d'un coup ce qu'était la musique, un peu tard je dois dire, mais enfin mieux vaut tard que jamais dans ce cas-là 39 . » Garant, somme toute largement autodidacte sur le plan de l'écriture, ne se reconnaîtra d'ailleurs plus tard qu'un seul maître : Messiaen ${ }^{40}$. Il écrira en 1978 :

À cause de la façon dont il enseignait, on devenait pénétré d'une certaine nécessité de construire la musique, de composer une musique à la fois sensible et intelligente, je dirais même intelligible, c'est-à-dire lisible sur le plan de la structure. Ce fut pour moi une véritable révélation ${ }^{41}$.

À peine remis du premier choc de la découverte de l'univers des Viennois, Garant est enthousiasmé par les nouvelles techniques de composition que

36 Serge Garant (1929-86) est né à Québec et sa famille se fixe ensuite à Verdun. À compter de 1941, il grandit à Sherbrooke où il commence son apprentissage musical. Voir Lefebvre, «Pour débusquer l'inconnu : chronologie de Serge Garant », Circuit 7, $\mathrm{n}^{0} 2$ (1996) : 57-73.

$37 \mathrm{Garant}$ demandera des cours privés de composition à Claude Champagne. Cet appel de la vocation aurait été fortifié par une audition bouleversante, sur disque, du Sacre du printemps de Stravinsky (entrevue accordée par Garant à Pierre Rolland et reproduite dans le coffret Anthologie de la musique canadienne : Serge Garant [Radio Canada International, ACM 2, 4 disques 33 1/3 tours, 1978]).

38 Voir les notes prises par Garant à la classe de Messiaen et conservées dans le Fonds Serge Garant, archives de l'Université de Montréal, document P. 141.

39 « Rencontre avec Serge Garant », interview de Maurice Fleuret, Cahiers canadiens de la musique, $\mathrm{n}^{\circ} 9$ (automne-hiver 1974) : 15, repris dans Lefebvre, Serge Garant et la révolution musicale au Québec, 74.

40Déclaration faite lors d'une rencontre publique avec Serge Garant animée par Maryvonne Kendergi le 20 mars 1972 à la Faculté de musique de l'Université de Montréal, et diffusée sur les ondes de Radio-Canada dans la série « Trésors d'archives de Maryvonne Kendergi » les 7 et 14 février 1992.

41 Hommage de Garant à Messiaen dans le dossier «Olivier Messiaen, $70^{\mathrm{e}}$ anniversaire », Variations $2, \mathrm{n}^{\circ} 2$ (novembre 1978): 8 . 
Messiaen propose. Les procédés de développement de motifs simples par l'ajout ou la soustraction de valeurs rythmiques, les modes d'attaques et de durée, les canons rythmiques indépendants de la mélodie et de l'harmonie, tout ceci prépare et conduit le jeune Serge Garant à la musique de Pierre Boulez ${ }^{42}$. Or, la filiation est directe puisque ce sont justement des pages de Messiaen comme l'étude pour piano intitulée Mode de valeurs et d'intensités qui viennent de fortifier Boulez et Stockhausen dans leur volonté d'élaborer une nouvelle esthétique musicale où le principe sériel est appliqué à tous les paramètres du son. Même si Messiaen n'épouse pas lui-même le sérialisme orthodoxe ni la cause pointilliste, il discute avec ses élèves de la fragmentation chez Webern, des beautés structurelles et rythmiques de la Deuxième sonate de Boulez (1947), de ses propres modes d'attaques et d'intensités. Il prône avant tout une pensée moderne, personnelle et structurée ${ }^{43}$. La classe d'analyse du Conservatoire de Paris peut donc être considéré comme le berceau du mouvement que l'on baptisera « sérialisme post-webernien » ou " généralisé ». Messiaen sera l'un des tout premiers professeurs européens à en rendre compte sur le plan historique et théorique, même avant Darmstadt. Le jeune Stockhausen est d'ailleurs l'un des confrères de Garant à la classe de Messiaen tandis qu'un Jean Barraqué en sortait tout juste ; quant à Boulez, après une période très critique à l'endroit de la musique de son ancien maître, il rend à l'occasion visite à celui-ci au Conservatoire et compose, en guise d'hommage, la première de ses Structures pour deux pianos (1951-52) à partir du mode de valeurs et d'intensités déjà mentionné. En grande partie grâce à Garant, qui sera à notre connaissance le premier à défendre la musique de Boulez au Canada, ce développement majeur de la pensée musicale européenne aura des répercussions beaucoup plus fortes au Québec que dans le reste du Canada.

En mai 1952, Garant revient au Québec « vraiment brûlant, tout feu, tout flamme », près à se battre pour des idées qu'il n'avait pas un an auparavant ; il n'est « plus le même être » ${ }^{44}$. À son tour, il ressent toutefois un violent décalage entre ce qu'il vient de vivre à Paris et la situation qui s'offre à lui au Québec, où tant reste à faire. Malgré les efforts louables de certains musiciens dévoués à la cause du développement culturel du Québec ${ }^{45}$, le public des concerts est

42Les premières compositions de Pierre Boulez seront à l'origine de l'autre profond bouleversement artistique éprouvé par Garant à Paris. Il reste complètement ébahi devant la complexité de la Sonatine pour flûte et piano (1946) et de la Deuxième sonate pour piano (1947). Andrée Vaurabourg-Honegger, dont il prend des leçons de contrepoint, l'aidera à vaincre son étonnement devant ce nouveau langage en lui montrant des exercices de contrepoint réalisé par le jeune Boulez au temps où celui-ci était son élève (propos de Garant, reproduits dans le coffret Anthologie de la musique canadienne : Serge Garant.

43Quoique Messiaen se soit généralement gardé de toute critique ouverte envers ses collègues compositeurs, ses élèves ont témoigné du fait qu'il laissait percevoir son peu d'intérêt pour toute forme de néo-classicisme et de conservatisme musical. Il préférait garder le silence sur une bonne portion de l'œuvre de Ravel, sur l'ensemble de la production du Groupe des Six, de Prokofiev, de Hindemith ou sur celle du Stravinsky d'après Les noces (voir Boivin, La classe de Messiaen, notamment 282-305).

44 « Rencontre avec Serge Garant », Cahiers canadiens de la musique, n" 9 (automne-hiver 1974) : 15, repris dans Lefebvre, Serge Garant et la révolution musicale au Québec, 74.

45 Il faut retenir ici le nom du chef d'orchestre Wilfrid Pelletier, membre fondateur et premier directeur artistique de l'Orchestre symphonique de Montréal (fondé en 1935), puis premier directeur du Conservatoire de musique de Montréal. 
encore trop rarement confronté à Brahms ou à Debussy. C'est dire que le mélomane moyen ne s'intéresse guère à la musique moderne, encore moins aux élucubrations de l'avant-garde ${ }^{46}$.

Mieux armé que Françoise Aubut face à ce profond hiatus culturel entre l'Ancien et le Nouveau Monde, Garant fait d'emblée preuve de leadership en ces années, pour reprendre les mots de Denise Leclerc, « de grand conformisme social et de conservatisme politique, [où] le monde de la culture était le seul refuge possible pour tout écart de pensée ${ }^{47}$. » Garant veut changer les choses. En octobre 1952, donc quelques mois après son retour, il présente avec le soprano Suzanne Gagnon à un auditoire sherbrookois un récital comprenant plusieurs pages de Messiaen, très vraisemblablement entendue en première québécoise sinon canadienne, nommément le cycle de mélodies Poème pour Mi (1937), le onzième des Vingt Regards sur l'Enfant-Jésus pour piano (« Première communion de la Vierge ») et l'île de feu I (tirée des Études de rythme). Le $1^{\text {er }}$ mai 1954 a lieu au Conservatoire de Montréal un concert que Lefebvre décrit comme la « première manifestation importante de musique contemporaine ${ }^{48}$ dans cette ville et auquel participe, outre Garant, ses amis François Morel et Gilles Tremblay. Le jeune Morel interprète en première canadienne deux des Études de rythme de Messiaen (Neumes rythmiques et Île de feu II), Tremblay joue trois extraits des Vingt Regards surl'Enfant-Jésus, tandis que Garant donne en première canadienne le mouvement initial de la Première sonate de Boulez (1946). La seconde partie du concert est consacrée à des œuvres des trois jeunes compositeurs. L'année suivante, à la même date et toujours au Conservatoire de Montréal, a lieu un autre concert mémorable placé sous le signe d'Anton Webern, dont plusieurs œuvres sont alors entendues pour la première fois au Canada. On présente également au public des compositions récentes de Garant, de Morel, de Gabriel Charpentier, d'Otto Joachim et de Jocelyne Binet ${ }^{49}$. En 1956, Garant fonde, en compagnie de ses amis Morel, Joachim et Jeanne Landry l'association « Musique de notre temps » qui, au cours de trois concerts (20

\footnotetext{
46 Au pays, au retour de Garant, la musique moderne se résume essentiellement aux noms de Hindemith, de Prokofiev et de Stravinsky. La musique des Viennois, de Messiaen et de Boulez est pour ainsi dire totalement inconnue (entrevue accordée par Garant à Pierre Roland, Anthologie de la musique canadienne : Serge Garant). Il est un fait que les concerts symphoniques sont encore à cette époque espacés et semi-professionnels. Si l'on fait exception de quelques événements isolés, comme le passage en tournée de solistes ou d'ensembles venus de l'étranger, il n'y a guère que par le disque ou la radio que l'on peut entrer parfois en contact avec un répertoire plus exigeant. Notons que Pierre Mercure, reconnu pour ses réalisations télévisées audacieuses à «L'heure du concert », n'entrera au service de la Société Radio-Canada qu'en 1952.

47Leclerc, La crise de l'abstraction au Canada, 37. On peut faire un rapprochement intéressant entre l'émancipation de la pensée picturale abstraite au Canada durant cette période et le développement de la pensée musicale sérielle, adoptée spontanément par Garant et certains de ses amis compositeurs. Dans ses écrits, Garant fait d'ailleurs souvent allusion à la peinture moderne et se plaint de ce que la musique québécoise accuse vis-à-vis d'elle un retard incompréhensible ; voir Denis Gougeon, " Garant communicateur ou la parole agissante », Circuit 7, $\mathrm{n}^{\circ} 2$ (1996) : 27-35 : voir aussi Lefebvre, Serge Garant et la révolution musicale au Québec, entre autres 106, 113).

48Lefebvre, Serge Garant et la révolution musicale au Québec, 48.

$49 \mathrm{Il}$ s'agit, dans le cas de Garant, de Nucléogamme pour bande magnétique et sept instruments (1955) et, dans celui de Binet, de la Pièce pour trio à cordes (sans date). Voir Lefebvre, Serge Garant et la révolution musicale au Québec, 46-48, pour le détail de la programmation de ces deux concerts-étapes.
} 
octobre 1957, 9 mars et 12 juillet 1958), fera découvrir au public montréalais trois œuvres de Messiaen, soit le Quatuor pour la fin du Temps (encore une fois vraisemblablement en création québécoise sinon canadienne), l'étude Mode de valeurs et d'intensités et le rondo Cantéyodjayâ (1949), ces deux dernières pages interprétées par la pianiste Josephte Dufresne.

En 1966, Garant devient directeur artistique de la toute nouvelle Société de musique contemporaine du Québec ${ }^{50}$. Le nom de Messiaen apparaîtra près de 20 fois sur les programmes des quelques 200 concerts donnés par la SMCQ jusqu'à la mort de Garant en $1986^{51}$. Au chapitre de la diffusion de l'œuvre de Messiaen, il faut encore ajouter les nombreuses retransmissions radiophoniques de ces concerts, les quelques recensions de disques rédigées par Garant entre 1959 et 1966 pour le Journal musical canadien et le Journal des jeunesses musicales du Canada, ainsi que de nombreuses présentations radiophoniques d'enregistrements sur disques dans le cadre de l'émission Musique de notre siècle, qu'il a animée de 1969 à 1985 sur les ondes de Radio-Canada. Enfin, soulignons que Garant analysait régulièrement les Quatre études de rythme de Messiaen dans son cours d'analyse à l'Université de Montréal. On s'en doute, ce sont les pages les plus « organisées » de Messiaen, celles composées justement au tournant des années 50 et au cours de la décennie suivante, qui intéressait surtout l'auteur des Offrandes.

Nous venons consciemment de bouleverser quelque peu la chronologie afin d'accentuer l'effet déclencheur de ce séjour d'études à Paris sur celui qui a été appelé à occuper l'avant-scène de la musique contemporaine québécoise pendant près de 40 ans à titre de compositeur, d'interprète, de directeur artistique de la SMCQ, d'animateur à la radio et de professeur d'analyse et de composition à l'Université de Montréal.

\section{Clermont Pépin et le marché parisien de la formation des compositeurs}

À l'opposé de Serge Garant, dont les premières connaissances en matière de composition et d'arrangement ont été acquises essentiellement à l'école de la musique populaire et du jazz, le jeune Clermont Pépin ${ }^{52}$ a été à cet égard très bien formé. D'abord un élève de Claude Champagne à titre privé (1939-41), il se perfectionne au Curtis Institute de Philadelphie (1942-45) alors que la guerre fait rage en Europe. Pépin retrouve ensuite Claude Champagne au Conservatoire de Montréal (1945-46) avant de « s'exiler » à nouveau durant trois années à Toronto pour y poursuivre ses études de piano et de composition. Lorsqu'il traverse finalement l'Atlantique en 1949, Clermont Pépin a 23 ans et est lauréat

\footnotetext{
50 Rappelons que quatre années auparavant, en 1962, Olivier Messiaen et Yvonne Loriod visitent pour la première fois le Québec ; invités par Clermont Pépin dans le cadre du « Festival de Montréal », ils interprètent les Visions de l'Amen pour deux pianos.

51 Les œuvres de Messiaen les plus souvent présentées par la SMCQ sont le Catalogue d'oiseaux (1958), Oiseaux exotiques (1958) et Couleurs de la Cité céleste (1963). Garant rédige souvent les textes de présentation des œuvres qu'il dirige et plusieurs de ces textes sont reproduits dans Lefebvre, Serge Garant et la révolution musicale au Québec. Le successeur de Garant au poste de directeur artistique de la SMCQ, Walter Boudreau, a tenu à poursuivre la tradition en organisant le 16 février 1989 un concert entièrement consacré à la musique de Messiaen.

52Clermont Pépin est né à Saint-Georges-de-Beauce en 1926.
} 
du Prix d'Europe. C'est le pianiste que l'on vient de récompenser, mais c'est surtout le jeune compositeur que la perspective de ce voyage rend fébrile.

$\mathrm{Au}$ Royal Conservatory de Toronto, Pépin avait formé le projet d'étudier la composition avec Arthur Honegger. Il fréquentera de fait durant trois ans sa classe à l'École normale. L'auteur du Roi David était déjà très malade - il devait mourir en 1955 - et, au dire de Pépin, ne s'intéressait que très peu à l'aspect technique des œuvres qu'on lui présentait ; il se bornait volontairement à des commentaires d'ordre assez général. Profondément pessimiste, il se montrait parfois assez dur avec ses élèves, au point de les inciter à ne placer aucun espoir dans la carrière de compositeur ${ }^{53}$. Le jeune Pépin pourra bientôt constater que l'atmosphère à la classe de Messiaen est très différente.

Pépin avait été mis en contact avec la musique de Messiaen à Toronto, mais les mélodies pour soprano et piano qu'on lui avait alors demandé de déchiffrer n'avaient guère éveillé son intérêt. Or, dès son arrivée à Paris, il entend parler de tous côtés, et en bien, de la classe d'analyse du Conservatoire et s'y rend à titre d'auditeur. Une photographie prise à la classe de Messiaen au printemps de 1951 nous montre, groupé autour de ce dernier assis au piano, une vingtaine d'élèves parmi lesquels nous reconnaissons Clermont Pépin, Sylvio Lacharité, Serge Garant, Yvonne Loriod et Karlheinz Stockhausen ${ }^{54}$. Il nous plaît d'imaginer les conversations réunissant de tels élèves autour d'un tel maitre !

Durant les six années qu'il vivra à Paris, Pépin côtoiera de près les réputés pianistes Lazare Lévy et Yves Nat, et bénéficiera des conseils d'Arthur Honegger ainsi que d'André Jolivet. Ceci nous amène à ouvrir une parenthèse pour préciser que si Messiaen a été le principal mentor des musiciens dont nous venons de dire quelques mots, la classe d'analyse du Conservatoire ne constituera en fait, pour plusieurs autres compositeurs québécois qui y séjourneront, qu'une étape parmi d'autres. Un apprenti-compositeur qui se rend dans la capitale française au début des années 50 peut en effet se tourner vers plusieurs maîtres respectés pour parfaire l'art de la composition. Tony Aubin et Darius Milhaud sont titulaires des classes de composition au Conservatoire, tandis que Honegger détient pour quelque temps encore une classe à l'École normale. René Leibowitz, Max Deutsch et Nadia Boulanger enseignent pour leur part l'écriture, essentiellement en privé ${ }^{55}$. Messiaen, s'il se distingue par le modernisme de sa pensée, est donc loin de détenir un monopole. Son influence sur le développe-

53On pourra comparer ce témoignage à celui, très similaire, de Xenakis (Boivin, La classe de Messiaen, 113).

54 La photographie est reproduite dans Lefebvre, Serge Garant et la révolution musicale au Québec, 44. Pépin n'a pu cependant nous préciser exactement à quel moment et combien de temps il a assisté aux cours de Messiaen.

55Une étude sur l'impact canadien de ces pédagogues renommés reste à faire. Notons simplement que Boulanger et Milhaud ont enseigné aux États-Unis et parlaient assez bien l'anglais ; tous deux étaient particulièrement en demande auprès des Américains de passage à Paris (Boulanger a pris en 1950 la direction du Conservatoire américain de Fontainebleau). À titre indicatif, on retiendra que István Anhalt, Françoise Aubut, Maurice Blackburn, Walter Buczynski, Gabriel Charpentier, Jean Coulthard, Andrée Désautels, Kenneth Gilbert, Irving Heller, Kelsey Jones, Peter Paul Koprowski, Jeanne Landry, Claude Lavoie, Pierre Mercure, Jean Papineau-Couture, Michel Perrault, Rosette Renshaw, William Keith Rogers, Paul Scherman et Calvin Sieb comptent parmi les nombreux élèves canadiens de Boulanger. Irving Glick a étudié auprès de Max Deutsch et, Bruce Mather, quant à lui, a été un élève de Milhaud. 
ment de plusieurs compositeurs québécois devra donc être « démêlée » de celle d'autres maîtres dotés de fortes personnalités et d'allégeances stylistiques fort diverses.

Revenons à Clermont Pépin. Même si sa musique a toujours été assez éloignée de celle de Messiaen, l'auteur de la Messe sur le monde juge rétrospectivement que de tous ses maîtres parisiens, c'est ce dernier qui l'a enrichi le plus. Il lui est par exemple reconnaissant de lui avoir fait découvrir la Suite lyrique d'Alban Berg, une œuvre sur laquelle Messiaen se serait attardé plusieurs semaines. "Maître, plus vous parlez de cette œuvre, plus je la déteste ! ", lui aurait déclaré un jour son jeune élève, qui réalise toutefois bientôt qu'une musique semblable ne révèle ses beautés qu'une fois qu'on en a pénétré l'esthétique et approfondi l'analyse. C'est à la suite d'un tel travail que Pépin a composé son Deuxième quatuor à cordes (1956), sa première œuvre entièrement sérielle, commencée à Paris et terminée à Montréal ${ }^{56}$.

Durant cette période, Messiaen a en outre analysé plusieurs de ses propres œuvres, comme La Nativité du Seigneur pour orgue (1935) - la séance s'est déroulée à la tribune de la Trinité -, la Turangalîla-Symphonie, le grand cycle de mélodies Harawi, chant d'amour et de mort (1945), des extraits des Vingt Regards sur l'Enfant-Jésus ainsi que les cruciales Études de rythme. Paraissant soucieux, comme tant d'autres élèves de Messiaen, de se distancier de cette production, Pépin a tenu à nous préciser qu'il a davantage retenu de celui-ci une méthode de travail que des traits stylistiques précis. C'est à l'« architecte rigoureux et passionné », au « Bach du $\mathrm{XX}^{\mathrm{e}}$ siècle » qu'il dit vouer en premier lieu de la reconnaissance ${ }^{57}$.

Pépin a d'abord été impressionné, tout comme l'a été Garant, par la preuve apportée par Messiaen qu'il est possible de « composer avec des chiffres sans que la palette sonore s'en trouve nécessairement réduite. » À cet égard, il loue l'imagination de l'auteur des Vingt Regards qui a su intégrer à son langage de fascinantes techniques de transformation de motifs simples, par exemple le développement par agrandissement ou rétrécissement d'intervalles. Les cours donnés par Messiaen sur les modes, et particulièrement sur ses propres modes à transposition limitée, ont également provoqué une réflexion féconde. Le troisième mode symétrique répertorié par Messiaen a été utilisé dans Guernica (1951), la première œuvre que Pépin reconnaît véritablement comme sienne, tandis que tel passage du Rite du soleil noir (1955) est écrit dans le deuxième mode, si caractéristique. Quant au légendaire Mode de valeurs, il a purement et simplement bouleversé le jeune compositeur : «Messiaen rejoignait Boulez par un autre chemin », des mots que nous avons aussi trouvés dans la bouche de Serge Garant ${ }^{58}$. Enfin, les longues séances consacrées par Messiaen au rythme ont porté fruit, qu'il s'agisse de la description détaillée des deçî-tâlas hindous

56Pépin a relaté cette anecdote à Gilles Potvin dans le cadre de l'interview gravée sur disque pour le coffret Anthologie de la musique canadienne : Clermont Pépin (Radio-Canada International, ACM 5, 4 disques $331 / 3$ tours, 1980).

57 Ces propos de Pépin sont extraits de l'entretien privé qu'il nous a accordé en février 1989.

58 « Propos sur Olivier Messiaen », émission mettant à l'honneur Serge Garant et Gilles Tremblay, diffusée à la radio FM de Radio-Canada le 22 août 1963 et conservée aux archives sonores de la Société. 
et des mètres grecs antiques, de l'analyse minutieuse des arsis et thesis du plain-chant ou encore de l'accentuation mozartienne. Pépin retient à cet égard un moment fort : l'analyse du Sacre du printemps de Stravinsky, sans doute la plus célèbre des analyses de Messiaen ${ }^{59}$. Les fameux « personnages rythmiques » découverts dans cette partition ont amené Pépin à concevoir ce qu'il appelle des " mélodies rythmiques ", des " rythmes ayant une personnalité mélodique ».

En somme, lorsqu'il est question de triturer des nombres, de composer avec des chiffres, Pépin reconnaît en Messiaen « un très grand génie ». Mais le discours du maître ouvrait sur bien d'autres univers. Pépin évoque d'intéressants apartés sur la composition micro-tonale, la théorie des harmoniques de Jacques Chailley, les idées de Ferruccio Busoni sur le total chromatique, le piano préparé de John Cage, la musique traditionnelle japonaise, les chants d'oiseaux, les rythmes aztèques ou l'art maya. On le voit, aucun sujet ne semblait à Messiaen trop ésotérique - « hors programme » comme on dirait aujourd'hui - dès lors qu'il s'agit de former des musiciens inventifs et à l'esprit ouvert.

\section{Roger Matton à l'écoute de deux maîtres}

Pour sa part, le jeune Roger Matton arrive à Paris au début de janvier 1950, soit un ans et demi avant Garant et quelques mois après Pépin. Il n'a pas encore 21 ans et fait la traversée en compagnie de son camarade Pierre Mercure ${ }^{60}$. Tout comme Clermont Pépin, Matton se nourrira en France à diverses tables. Il sera l'élève d'Andrée Vaurabourg-Honegger — en privé puis à l'École normale -, ainsi que celui de Messiaen et de Nadia Boulanger, parfois concurremment.

Peu après qu'un ami l'ait recommandé à Boulanger, Matton se voit aux prises avec un délicat conflit d'horaire. En effet, les cours de Messiaen sont toujours très longs (de 16 heures à 20 heures, trois fois par semaine), tandis que Boulanger tolère difficilement l'absence aux siens. De plus, l'orientation esthétique de ces deux personnalités exceptionnelles se révèle fort différente - et complémentaire - puisque Boulanger donne en exemple à ses élèves les grands contrapuntistes de la Renaissance, les madrigaux de Monteverdi et les chefsd'œuvre de Bach alors que Messiaen, qui se déclare avant tout un harmoniste et un rythmicien, se réclame plutôt de Debussy ; la stabilité métrique de Bach le lasse et il n'éprouve aucun intérêt pour le néo-classicisme défendu par sa collègue, préférant nettement les premiers ballets révolutionnaires composés par Stravinsky pour Diaghilev à sa production plus récente ${ }^{61}$. Il semble par

59 On pourra lire les quelques pages que nous avons consacrées ailleurs à cette analyse (Boivin, $\mathrm{La}$ classe de Messiaen, 282-95) et surtout le passage important qui y est consacré dans le deuxième tome du Traité de rythme, de couleurs et d'ornithologie de Messiaen (Paris : Leduc, 1995), 93-147, un ouvrage posthume monumental dont deux tomes sont parus au moment d'écrire ces lignes.

60 Quoique Mercure ait fait, d'après Roger Matton, au moins une visite à Messiaen au Conservatoire, le futur organisateur de la Semaine de musique actuelle de Montréal (1961) s'est d'emblée dirigé du côté de Pierre Schaeffer et de son Studio d'essai à la radio. Mercure s'intéresse déjà à l'improvisation collective, de même que son ami Gabriel Charpentier, élève de Nadia Boulanger.

61 Parmi les autres compositeurs particulièrement appréciés de Messiaen, mentionnons encore Mozart, Berlioz, Wagner, Moussorgsky et Albéniz. 
ailleurs que si Boulanger respecte en Messiaen le musicien et l'organiste accomplis, elle ne peut supporter sa musique.

L'expérience de Matton à la classe de Messiaen se divise en deux temps. Il assiste aux cours d'analyse d'abord en 1950, l'année de son arrivée, de façon assez assidue. C'est Jocelyne Binet qui le présente au Maître ${ }^{62}$. Ce jour-là, Messiaen analyse un concerto de Mozart, et Matton est complètement emballé. Il n'hésite pas à déclarer que la démarche analytique de Messiaen "vaut n'importe quel cours de composition ». Il voit en celui-ci « un guide».

Matton connaissait un peu la musique d'orgue de Messiaen avant son départ pour la France ${ }^{63}$, mais s'intéressait alors plutôt à l'œuvre de Hindemith. Le langage si personnel de Messiaen lui devient familier autant par le biais des analyses que celui-ci propose de sa propre musique (par exemple les Poèmes pour $M i$, décortiqués durant plusieurs semaines) que par l'étude du traité Technique de mon langage musical (1944) que Matton se hâte d'acquérir. Le jeune musicien en vient tout naturellement à " parler en mode 2 », le plus caractéristique des modes définis par Messiaen. Sur la musique du compositeur français, Matton a toutefois aujourd'hui des opinions ambivalentes, rejoignant en ceci plusieurs autres élèves, toutes nationalités confondues. D'abord séduit par La Nativité du Seigneur (1935), il qualifie de "géniale » la fin de L'Ascension (1933), avec ses accords lents et somptueux, et considère le Quatuor pour la fin du Temps comme l'une des plus grandes œuvres de Messiaen. Chronochromie (1960), une œuvre synthèse où l'on trouve concentré l'essentiel des préoccupations de celui qui se décrivait comme compositeur, ornithologue et rythmicien, occupe dans son univers musical une place privilégiée. Par contre, il affirme avoir toujours détesté la Turangalîla-Symphonie; le Mode de valeurs, pour sa part, lui semble plus courageux qu'émouvant.

Matton n'a pas conservé beaucoup de musique composée à l'époque de ses études à Paris, ce qui peut expliquer pourquoi l'influence de Messiaen n'est pas très sensible à l'audition de ses premières œuvres reconnues, marquées plus directement par les langages de Stravinsky, de Bartók et par les emprunts au folklore québécois. Car l'avenir nous montrera qu'une fois conquises les difficultés de l'écriture musicale, l'influence de Nadia Boulanger va dominer. Malgré tout, Matton dit s'être laissé imprégner par certains éléments du langage de Messiaen, que ce soit au chapitre de l'harmonie, du rythme ou du timbre instrumental. Il croit lui devoir surtout un encouragement à " colorer " son écriture, à la libérer des modèles traditionnels. Il juge que l'insistance de Messiaen sur les chants d'oiseaux et les mélodies du plain-chant l'a aidé à se libérer des entraves de la barre de mesure et à développer une écriture mélodique mélismatique, détachée du mètre. Notons que Matton se servira de thèmes

62 À preuve que les musiciens québécois exilés volontairement à Paris constituent une petite communauté, on notera que Binet est alors une amie de la pianiste Raymonde Gagnon, depuis peu l'épouse de Pépin. Le couple a d'ailleurs joué les deux premiers mouvements du Concerto pour deux pianos et percussion de Matton (1955) sur les ondes de la radio française. Tous ces Québécois, auxquels s'ajoutent encore Garant et Lacharité, pouvaient se croiser à l'occasion chez Messiaen.

63Matton a touché l'orgue à l'occasion et, une fois à Paris, il se fait un devoir d'aller aussi souvent que possible entendre Messiaen improviser à La Trinité. 
grégoriens dans sa Suite de Pâques pour orgue (1952), composé entre les deux séjours à la classe d'analyse de Messiaen.

À ce propos, il convient de préciser que Matton n'a jamais montré ses travaux de composition à Messiaen, pas plus que ne l'ont fait Garant ou Pépin. Rappelons qu'à l'époque qui nous occupe, Messiaen n'enseigne pas encore la composition, mais bien l'analyse, même s'il aborde celle-ci en compositeur "pratiquant "64. Dans le cas des trois élèves que nous venons de nommer, l'influence du Maître est donc indirecte ; elle passe avant tout par l'analyse des cuvres, anciennes ou modernes.

Dans l'hommage écrit qu'il rendait en 1978 à son ancien maître, Matton souligne encore le fait que Messiaen était le seul à pouvoir à cette époque offrir à ses élèves une aussi vaste connaissance du répertoire contemporain ${ }^{65}$ et à leur permettre de côtoyer les étoiles montantes de la musique d'avant-garde que sont les jeunes Boulez, Stockhausen, Cage, Barraqué et Xenakis, tous rencontrés chez Messiaen. Pour finir, l'auteur de L'Escaouette insiste sur la beauté et l'efficacité du verbe de Messiaen, sur la force que peut prendre, lorsqu'elle est ainsi maîtrisée, la langue française comme outil de description de la musique.

\section{Un ensemencement en terre fertile}

Au cours de ces années cruciales, une transformation du milieu musical québécois est en cours. La Ligue des compositeurs présidée par Jean Papineau-Couture organise, en association avec d'autres institutions, des concerts de musique canadienne. Indéniablement, un nouveau public se crée, éduqué et sensibilisé à la nécessité de soutenir le développement d'une culture québécoise distincte. Cet éveil d'intérêt pour la musique nouvelle, nourri par un nationalisme grandissant et un meilleur soutien institutionnel (bourses, commandes, concours, productions radiophoniques, etc.), engendre un regain d'énergie créatrice chez les compositeurs. Le nouveau Quatuor de Montréal, composé notamment des frères Otto et Walter Joachim, inaugure ses activités en mars 1955 par un récital consacré exclusivement à des œuvres canadiennes ; c'est cet ensemble qui crée en 1957 le Deuxième quatuor de Pépin.

Pour le jeune Matton, comme pour Pépin, le séjour à Paris aura été fructueux, à plusieurs titres. Le premier, après avoir effectué quelques aller-retour entre la France et le Québec, revient définitivement au Canada en 1955 et s'installe à Québec à la fin de la même année. Une nouvelle carrière d'ethnomusicologue, encouragée par le vétéran Marius Barbeau, l'éloigne a priori des conceptions plus abstraites d'un Messiaen. Matton n'en poursuit pas moins son cheminement de compositeur. Il a par ailleurs enseigné quelques années la composition et l'histoire de la musique contemporaine (1960-63) à l'Université Laval et s'est fait un plaisir d'accueillir son ancien maître Messiaen lors de son passage à Québec en 1970.

64Lorsqu'on le lui demandait, Messiaen acceptait toutefois de bonne grâce de jeter un coup d'oeil sur une partition à la fin de sa classe.

65 Dossier "Olivier Messiaen, $70^{\mathrm{e}}$ anniversaire ", Variations 2, $\mathrm{n}^{\circ} 2$ (novembre 1978) : 10. Ce document comprend aussi, parmi d'autres témoignages d'élèves québécois de Messiaen, celui de Clermont Pépin. 
Pépin rentre lui aussi au Québec en 1955, emportant sous son bras de nouvelles partitions, mais aussi Technique de mon langage musical, dont il va se servir à des fins pédagogiques, car il est aussitôt nommé professeur de composition et d'écriture au Conservatoire de Montréal (1955-64) par Wilfrid Pelletier ${ }^{66}$. Dès lors, Pépin parle souvent à ses élèves de Messiaen et puise dans son traité de nombreux exemples pédagogiques d'une syntaxe musicale véritablement contemporaine.

Un nouveau maillon de la chaîne vient de se former. Au moins trois des élèves de Pépin au Conservatoire vont en effet bientôt se rendre à leur tour à Paris et, de là, chez Messiaen. Il s'agit d'André Prévost, de Jacques Hétu et de Micheline Coulombe Saint-Marcoux. Les deux premiers séjourneront chez Messiaen au début des années 60 et en seront assez profondément marqués. Pour sa part, Coulombe Saint-Marcoux, lauréate du Prix d'Europe de composition en 1967, n'effectuera, semble-t-il, qu'un saut à la classe de Messiaen, devenue en 1966 une classe de composition en titre ${ }^{67}$; elle s'orientera plutôt vers le Groupe de Recherches Musicales (G.R.M.) dirigé par Pierre Schaeffer et vers la classe de ce dernier au Conservatoire de Paris.

Revenons maintenant quelques années en arrière afin de retrouver un autre jeune musicien québécois qui vient lui aussi de réaliser un rêve et de mettre à son tour le pied dans Paris.

\section{Gilles Tremblay, le fils spirituel}

Le compositeur québécois que l'on associe le plus spontanément à Messiaen est certainement Gilles Tremblay. Il a maintes fois interprété sa musique, a souvent rendu hommage oralement ou par écrit à l'homme, au pédagogue et au compositeur et est demeuré l'un de ses proches jusqu'à la mort de celui-ci en $1992^{68}$.

À compter de l'automne 1954, alors que Paris est en pleine effervescence sérielle, Tremblay assiste assidûment à la classe d'analyse du Conservatoire. Tandis que ses compatriotes l'ont fait généralement à titre d'auditeurs, il y reste trois années complètes à titre d'étudiant étranger, le temps d'obtenir son prix ${ }^{69}$.

66 Pépin devient ensuite directeur des études puis accède à la direction du Conservatoire (1967-72). Il reprendra l'enseignement de l'écriture et de la composition en 1972 aux conservatoires de Montréal et de Québec.

67 Chose certaine, Messiaen a commenté à sa classe de composition le 9 octobre 1968 les Séquences pour deux Ondes Martenot et percussion de Coulombe Saint-Marcoux, une œuvre terminée en cette même année, ce qui nous incite à croire que la jeune compositrice aurait été autorisée à assister à la classe du maître à titre d'auditrice (notes prises cette année-là par sœur Blanche Leblanc (voir Boivin, La classe de Messiaen, 443-46).

68Retenons à ce propos, outre la participation de Tremblay en tant qu'ondiste à la première canadienne de la Turangalîla-Symphonie à Toronto en 1964, la version intégrale, en deux soirées, du vaste Catalogue d'oiseaux, présentée en équipe avec le pianiste Gilles Manny en août 1967, au pavillon du Canada de l'Exposition universelle de Montréal, dans le cadre des concerts de la SMCQ. Au chapitre des écrits, soulignons l'article élaboré intitulé " Oiseau-nature, Messiaen, musique ", Les Cahiers canadiens de musique, printemps-été $1970: 15-40$. On consultera la liste des articles, entrevues et commentaires radiophoniques rassemblés dans le numéro "Gilles Tremblay : réflexions », Circuit 5, n 1 (1994) : 73-76.

69 Outre Françoise Aubut (pour la classe d'harmonie) et Gilles Tremblay, les seuls élèves canadiens de Messiaen à avoir été officiellement inscrits à sa classe sont Talivaldis Kenins (letton d'origine), Bruce Mather, Jacques Hétu et Steven Gellman. Les autres n'ont donc pas eu à traverser l'épreuve du concours 
Parallèlement, il étudie le piano avec Yvonne Loriod, depuis plusieurs années l'interprète privilégiée de Messiaen. Loriod prête parfois main forte à Messiaen lorsque celui-ci analyse à sa classe des pièces du répertoire pianistique qu'elle connaît bien et qui sont particulièrement exigeantes sur le plan technique, par exemple des concertos de Mozart, Iberia d'Isaac Albéniz ou encore les différents volets du vaste Catalogue d'oiseaux (1956-58) que Messiaen présente à ses élèves au fur et à mesure qu'il en termine la composition. Cette invitation du musicien à pénétrer librement dans son " jardin secret », pour reprendre l'expression de Tremblay, et à observer de près son processus de création sera perçue, par ce dernier et plusieurs de ses compagnes et compagnons de classe, comme un rare privilège et la marque d'une grande générosité artistique.

Il faut bien dire qu'au fil des ans, depuis ses débuts de professeur au Conservatoire, Messiaen a acquis une stature internationale. Ses œuvres sont maintenant jouées régulièrement en France, notamment aux concerts du Domaine musical. Il compte, avec Boulez, parmi les compositeurs français vivants les plus connus en dehors de l'Hexagone ${ }^{70}$. Sa renommée de pédagogue s'est elle aussi répandue rapidement, soutenue par la fulgurante carrière $\mathrm{d}$ 'anciens élèves - Boulez, Stockhausen et Xenakis en tête - qui le reconnaissent comme seul véritable maître à penser. En un mot, Messiaen est devenu célèbre et ses élèves sont désormais conscients, en venant à sa classe, d'inscrire leurs noms dans une page importante de l'histoire musicale occidentale.

Dans ce contexte nouveau, plusieurs facteurs font de Gilles Tremblay un cas à part parmi les élèves canadiens de Messiaen. D'une part, une communion d'esprit s'établit très vite entre le maître et l'élève sur plusieurs points fondamentaux, notamment la spiritualité chrétienne, si importante dans leurs démarches respectives. D'autre part, Tremblay a pu bénéficier d'un contact personnel suivi avec Messiaen sur le plan de l'apprentissage de la composition proprement dite. Il s'est souvent rendu chez lui afin de lui montrer ses travaux récents, une habitude qu'il a conservée longtemps après sa sortie de la classe, à chaque fois que l'occasion se présentait. À l'exception peut-être de Françoise Aubut, aucune des personnes dont il a été question jusqu'ici ne semble avoir profité de cette occasion d'entendre, ponctuellement ou sur une base régulière, les conseils de cette sommité mondiale sur leurs travaux en cours ${ }^{71}$.

Interrogé sur cette expérience unique, Tremblay insiste aujourd'hui sur la grande simplicité de l'homme et du compositeur, sur l'absence totale de volonté de puissance du pédagogue sur ses élèves. Il parle de Messiaen comme d'un révélateur, d'un catalysateur qui a mis en mouvement des forces vives déjà présentes en lui. L'auteur de Solstices ne partage-t-il pas avec celui du Réveil des oiseaux une réelle fascination pour les phénomènes naturels, le bruissement du vent, le cycle des saisons ? Sa musique et ses propos ne témoignent-ils pas

de fin d'année.

70 Ajoutons à ces deux noms celui de Francis Poulenc, alors âgé et qui évolue dans une toute autre sphère.

71 Jacques Faubert et Steven Gellmann auront plus tard, à la classe de composition de Messiaen au Conservatoire (Gellmann y est inscrit de 1973 à 1976), un accès direct à l'ample expérience professionnelle de celui-ci. 
d'un même désir de célébrer la grandeur de la création divine, d'une ouverture aux musiques extra-européennes et aux philosophies orientales, de préoccupations pour le phénomène de la durée, pour le rythme inhérent à toute chose ? Plusieurs des titres donnés par Tremblay à ses œuvres, même récentes, semblent répondre à ceux qui figurent au catalogue de Messiaen : Cantique de durées, Compostelle I, "...le sifflement des vents porteurs de l'amour... », Vers le soleil, Vêpres de la Vierge, Musique du feu, Vers une étoile, etc. Tremblay a par ailleurs lui-même souligné l'importance de l'analyse du recueil pour piano Iberia d'Albéniz sur son évolution de compositeur ; l'impact stimulateur est particulièrement sensible, à son avis, dans l'écriture de Phases et Réseaux, deux pièces pour piano (1956-58) « où tout est exubérance et hyperacciaccaturé »72.

Quoique Gilles Tremblay se soit également perfectionné en France auprès d'autres musiciens de haut calibre (tels Maurice Martenot, Andrée VaurabourgHonegger, Karlheinz Stockhausen, Pierre Schaeffer, Pierre Boulez et Henri Pousseur), et en dépit du fait qu'il se dise gêné par cette association forcément quelque peu réductrice, nous ne pouvons nous empêcher de voir en lui un véritable fils spirituel de Messiaen, dans le sens le plus positif de l'expression. Certes, il a su se forger un style personnel de composition, mais ses idéaux demeurent très près de ceux de son défunt maître et ami. Comme lui, il aura recours aux beautés discrètes et éternelles du plain-chant, aux sonorités uniques des Ondes Martenot, aux ressources multiples des instruments de percussion. Les cours d'analyse et de composition qu'il a donnés à compter de 1961 aux conservatoires de musique de Québec et de Montréal s'inscrivent indéniablement dans la continuité de ceux de Messiaen au Conservatoire de Paris ${ }^{73}$. Les mêmes thèmes y ont été abordés, les mêmes œuvres analysées - y compris un bon nombre signées par Messiaen - dans un semblable esprit de syncrétisme, voire d'œcuménisme musical. La poésie du langage analytique de Tremblay est également largement tributaire du style de l'auteur du Traité de rythme, de couleur et d'ornithologie ${ }^{74}$. Enfin, notons que Tremblay a été impliqué dès 1968 dans les activités de la SMCQ et a donc vivement encouragé la diffusion des œuvres de Messiaen par cette importante société de concerts.

Terminons ce survol en insistant sur le fait que trois autres représentants importants de cette riche génération de compositeurs québécois se retrouveront au début des années 60 sur les bancs de la classe de Messiaen : André Prévost, Jacques Hétu et Bruce Mather. Tandis que Mather suivra parallèlement la classe de composition de Milhaud, Hétu et Prévost étudieront avec Henri Dutilleux. Tous trois ont évoqué pour nous avec enthousiasme cette période de leur formation ${ }^{75}$. Depuis le milieu des années 50 , le passage chez Messiaen est pour

72 Communication personnelle du compositeur. Yvonne Loriod assurera la création de ces pages en 1959 au Westdeutscher Rundfunk (Cologne).

73 Tremblay a enseigné plus précisément l'analyse au Conservatoire de musique de Québec de 1961 à 1966, ainsi que l'analyse et la composition au Conservatoire de Montréal de 1962 à 1996.

74 Voir le numéro de Circuit 6, $\mathrm{n}^{\circ} 1$ (1995) consacré aux analyses de Tremblay (« Tremblay/Varèse/Messiaen : Tremblay analyste ») et tout particulièrement l'article intitulé « Silences, silence » qu' on pourra comparer au ton général du Traité de Messiaen, lequel a été rédigé à partir du matériel accumulé par celui-ci pour ses cours au Conservatoire.

750n pourra trouver des témoignages en ce sens dans Boivin, « La classe de Messiaen : historique, 
ainsi dire devenu une tradition chez les jeunes compositeurs québécois. Si l'on examine cependant la chose d'un point de vue global, l'influence à long terme du célèbre musicien français aura été plus significative au cours de la décennie que nous venons de parcourir. Par la suite, les moyens de déplacement et d'accès à l'information - musicale, culturelle ou autre - seront tout autres et l'apport de Messiaen s'en trouvera relativisé.

\section{Une fenêtre ouverte sur la musique de toute allégeance}

Certes, les trajectoires artistiques que nous venons de résumer en quelques traits rapides sont distinctes et ne peuvent se ramener au simple dénominateur commun d'une rencontre avec Olivier Messiaen. Mais il demeure que les compositeurs canadiens-français qui résident à Paris au cours des années 50 observent des premières loges les bouleversements profonds qui agitent alors l'avantscène musicale européenne. Or, cette conjoncture aura des effets directs et déterminants sur la nouvelle musique qui s'écrira au Québec, notamment sur le plan de la structuration du langage musical et, comme le remarquait à juste titre Clifford Ford, sur celui de l'instrumentation, où domine souvent les instruments à percussion ${ }^{76}$. C'est généralement Messiaen qui fait office de porte d'entrée vers ce nouvel univers sonore. En vérité, ce que nous avons appelé la « filière québécoise » est à notre avis unique lorsque l'on examine le rayonnement global de Messiaen à l'extérieur de la France ${ }^{77}$. Les compositeurs québécois se relaient pour ainsi dire à sa classe, s'avisent les uns les autres de l'importance de ce qui s'y passe, s'associent pour jouer sa musique et diffuser ses idées en matière de composition.

On revient en Amérique en emportant sous le bras des partitions de Messiaen et des exemplaires de son credo en matière artistique, Technique de mon langage musical, dont on se servira comme alternative aux traités académiques en faveur par exemple au Conservatoire de Montréal. Un réel enseignement de la composition vient de naître au Québec français, un enseignement qui va dorénavant plus loin que les traditionnels exercices de pastiches de chefs-d'œuvre qui n'incitaient guère, au dire de Garant, au développement d'une personnalité originale. Au même moment se forment à Montréal des structures de diffusion d'une musique véritablement moderne, celle que l'on importe d'Europe (les œuvres de Webern, de Messiaen, de Boulez), mais aussi celle que composent la

reconstitution, impact ». À titre d'exemple, Mather, compositeur anglophone ayant des attaches très fortes avec la culture française, s'est révélé un excellent ambassadeur des idées rythmiques de Messiaen à l'Université McGill. Pour sa part, séduit par l'humanisme de Messiaen qui rejoint des cordes sensibles, Prévost sera plutôt marqué par l'esthétique de Dutilleux, le cadet de huit ans de Messiaen.

76Clifford Ford, Canada's Music : An Historical Survey (Toronto : Agincourt, 1982), 235. Ford signale aussi à ce propos l'utilisation que font certains compositeurs québécois des Ondes Martenot, instrument français par excellence et très apprécié par Messiaen au début de sa carrière.

77 Il va de soi que la question de la langue maternelle explique en partie cette filiation étroite et particulière entre l'auteur du Livre d'orgue et les compositeurs québécois qui ont été formés dans l'après-guerre. Messiaen ne parlait ni ne comprenait bien l'anglais, ce qui limitera considérablement la portée de sa musique et de sa pensée au Canada anglais ; de plus, ses référents sont puisés avant tout dans la culture française, donc moins signifiants pour de jeunes musiciens anglophones, à plus forte raison s'ils sont de confession protestante. 
jeune génération des Serge Garant, François Morel et Gilles Tremblay dans la volée de la découverte et de l'étude approfondie de ces nouveaux langages. Qu'on apprécie ou non la musique de Messiaen pour ses valeurs expressives, c'est bien elle qui servira de point de contact entre trois courants fondamentaux : la grande tradition française, la Seconde École de Vienne et l'esthétique boulezienne. De plus, la constance de la présence québécoise chez Messiaen favorisera les échanges stimulants entre les compositeurs de cette partie du pays et une pléiade de jeunes musiciens et musiciennes qui fréquentent sa classe au même moment, qu'ils aient pour noms Yvonne Loriod, Pierre Boulez, Marius Constant, Iannis Xenakis, Karlheinz Stockhausen, Makoto Shinohara ou Gilbert Amy, tous liés avec le Québec ${ }^{78}$.

Il serait imprudent d'attribuer tous les développements de la vie musicale québécoise au seul Messiaen et à ses idées, aussi originales fussent-elles. L'organisation d'une milice d'avant-garde à Montréal était inéluctable, comme le montrent des percées semblables à Toronto et ailleurs au Canada. On peut cependant affirmer qu'au Québec, les circonstances étaient particulièrement favorables à la consolidation rapide d'un modernisme radical, inspiré de celui que proposent en sol français les Messiaen, Boulez et autres. Le retard était, il faut bien le dire, considérable. Des compositeurs comme Serge Garant - et, dans une autre orientation esthétique, Pierre Mercure - ont mis les bouchées doubles pour faire connaître et accepter au pays la musique la plus iconoclaste.

L'essentiel de la pensée de Messiaen a été transmise jusqu'à récemment au Conservatoire de Montréal par Gilles Tremblay (et, avant cela, au Conservatoire de Québec). À divers degrés, elle l'a été aussi, ou continue de l'être, par d'autres compositeurs-pédagogues répartis dans la plupart des grandes institutions d'enseignement : André Prévost à l'Université de Montréal ; Bruce Mather, Bengt Hambraeus et Alcides Lanza à l'Université McGill ; Jacques Hétu à l'Université du Québec à Montréal ; Jacques Faubert au conservatoire de cette même ville. À Québec, un François Morel, l'un des rares compositeurs de cette génération à ne pas avoir été en mesure de se perfectionner en Europe, n'hésite pas à reconnaître sa dette envers l'auteur des Études de rythme.

On aura noté que ces noms ne forment guère une école homogène, et ceci vaut qu'on s'interroge. Au cours des années 50, alors que le discours dominant sur la création musicale s'appuie essentiellement sur l'intellect, Messiaen enseigne non pas à partir du tableau noir, accessoire obligé des sériels, mais plutôt assis à son piano, en s'appuyant sur le son direct et les partitions de grands compositeurs. Il parle d'harmonies colorées, de sonorités instrumentales chatoyantes, de mélodies souples conçues sur le modèle du plain-chant, de la beauté et de la variété des chants d'oiseaux, autant que de données plus abstraites comme les rythmes non rétrogradables et les modes de durées. Le point est

78 La programmation de la SMCQ, de même que les visites organisées par les institutions d'enseignement comme le Conservatoire de Montréal ou les facultés de musique des universités McGill ou de Montréal témoignent de ce réseau amical. On notera par exemple que Makoto Shinohara, compositeur japonais qui a séjourné au Québec à plusieurs reprises à titre de professeur invité à l'Université McGill, a été un élève officiel de Messiaen en 1956-57, au même moment que Gilles Tremblay (et possiblement un auditeur au cours des deux années précédentes). 
important. Tremblay dira qu'à la classe de Messiaen, les véritables maîtres étaient les compositeurs dont les œuvres se succédaient sur le piano de l'analyste : Debussy, Stravinsky, Chopin, Wagner, et tant d'autres encore. En pleine hégémonie sérielle, Messiaen, pour reprendre l'expression de Tremblay, « ouvrait une fenêtre sur la musique » et ce, pour le plus grand profit de musiciens de toutes allégeances. Là réside sans nul doute l'une des forces majeures de son intervention pédagogique.

Il n'aura été question ici que des compositeurs. Si l'on ajoute à la liste des élèves québécois de Messiaen les interprètes et les pédagogues de carrière, les communicateurs, les étrangers qui ont séjourné au Québec à titre de professeurs invités, le réseau se ramifie et se complexifie considérablement, du côté francophone comme au Canada anglais ${ }^{79}$. C'est pour célébrer cette influence constante de Messiaen sur l'évolution de la musique québécoise que la SMCQ a organisé le 30 octobre 1978 un concert-anniversaire, à l'occasion duquel on a demandé à un bon nombre d'anciens élèves du compositeur français un court texte relatif à leur expérience ${ }^{80}$. On a invité d'ailleurs ceux d'entre eux qui étaient présents à monter sur la scène de la salle Pollack de l'Université McGill afin de prendre part à la remise de la médaille du Conseil canadien de la musique présentée à Messiaen par la présidente du Conseil, Maryvonne Kendergi. Le geste était plus qu'honorifique. Il était pleinement justifié.

79 La liste est longue, mais on peut penser par exemple au cas de la chanteuse et pédagogue Josèphe Colle, passionnée d'analyse et auditrice à la classe de Messiaen en 1955-56, qui a fait partie de la première équipe des interprètes fidèles de la SMCQ, y a créé plusieurs œuvres des compositeurs québécois nommés ici et qui a enseigné dans diverses institutions au Canada et à l'étranger. Quant au musicologue Ramón Pelinski, il a utilisé les notes sur le rythme prises chez Messiaen dans ses cours à l'Université de Montréal. 80SMCQ, programme $n^{0} 109$ (30 octobre 1978) : 19-26. Le programme contient des témoignages de Françoise Aubut, Serge Garant, Roger Matton, Clermont Pépin, Sylvio Lacharité, André Prévost, Bruce Mather, Steven Gellman, Gilles Tremblay, Jacques Hétu, Ramón Pelinski et Aline Legrand. À ce document s'ajoutent les témoignages recueillis pour le dossier "Olivier Messiaen, $70^{\mathrm{e}}$ anniversaire » publié dans Variations $2, \mathrm{n}^{0} 2$ (novembre 1978) : 5-11, 19, 31. 


\section{Appendice : La présence québécoise et canadienne chez Messiaen}

\section{Cours privés}

1938

1939

1940

1941

1942

1943

1944

1945

1946

1947

1948

1949

1950

1951

1952

1953

1954

1955

1956

1957

1958

1959

1960

1961

1962

1963

1964

1965

1966

Françoise Aubut (organiste et compositrice)

(internement de Messiaen au stalag)

1

Classe d'harmonie (et cours privés)

Françoise Aubut (inscrite, 1941-44)

Jean-Louis Martiner (cours privés)

(visites)

\section{Classe d'analyse}

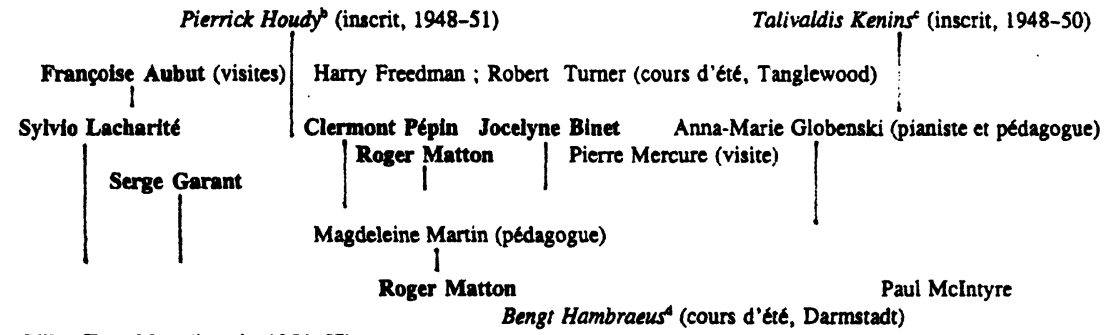

Gilles Tremblay (inscrit, 1954-57)

Bengt Hambraeus's (cours d'été, Darmstadt)

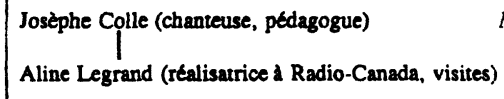

Aline Legrand (réalisatrice a Radio-Canada, visites)

Makoro Shinoharae (auditeur, puis inscrit en 1956-57)

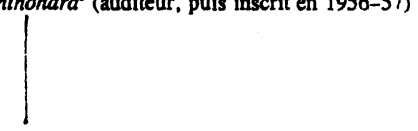

Bruce Mather (visiteur occasionnel, puis inscrit en 1960-61)

André Prévost

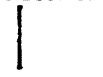

I (auditeur occasionnel)
Ramón Pelinski' (musicologue, pédagogue, pianiste)

1

Jacques Hétu (inscrit, 1962-63)

Alcides Lanza' (cours d'été, Buenos Aires) 


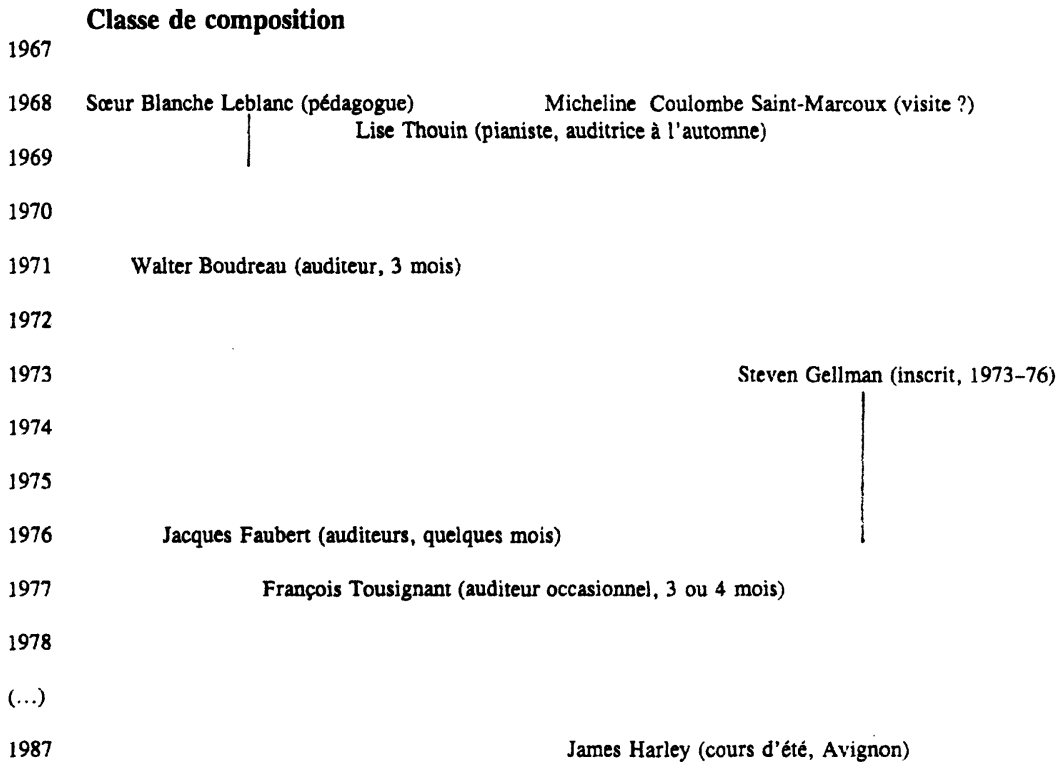

\footnotetext{
'Compositeur français né en 1912, professeur invité au Conservatoire de Montréal de 1971 à 1976.

'Compositeur français naturalisé canadien, né en 1929, professeur d'écriture et de composition à l'Université Laval (1970-71) puis au Conservatoire de Québec à partir de 1971. Il est retraité depuis 1992 et habite maintenant en France.

'Compositeur et pédagogue d'origine lettonne, né en 1919 et établi au Canada depuis 1951. Il a enseigné de 1952 à 1984 à l'Université de Toronto et est devenu directeur des classes de composition en 1977. Bruce Mather et Steven Gellman comptent parmi ses éleves.

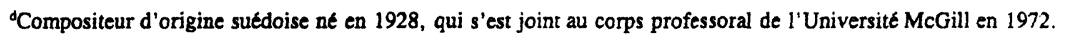

'Compositeur japonais, invité à plusieurs reprises par l'Université McGill à titre de professeur invité.

'Musicologue et pianiste d'origine argentine né en 1932, qui a enseigné à l'Université d'Ottawa de 1973 à 1977 puis, de 1977 à 1996, à l'Université de Montréal.

'Compositeur, chef d'orchestre et pianiste d'origine argentine, né en 1929, professeur à l'Université McGill depuis 1971. Il a été nommé directeur du studio de musique électronique en 1974.
}

\section{Résumé}

À sa classe du Conservatoire de Paris, Olivier Messiaen a enseigné l'harmonie, l'analyse musicale ou la composition à un nombre important de musiciens et de compositeurs canadiens, pour la plupart nés ou vivant au Québec. Sa musique et ses idées ont eu un impact réel sur la création musicale d'avant-garde au Québec ainsi que sur l'enseignement de la composition surtout au cours des 15 années qui ont suivi la Seconde Guerre mondiale. Plusieurs des élèves québécois de Messiaen (par exemple Serge Garant, Clermont Pépin et Gilles Tremblay), sont devenus à leur tour des pédagogues et ont contribué à faire connaître ses œuvres et sa pensée. 